\title{
Global Publications Output in Quantum Computing Research: A Scientometric Assessment during 2007-16
}

\author{
S. M. Dhawan ${ }^{\mathrm{a}}$, B.M.Gupta ${ }^{\mathrm{b}}$, Sudhanshu Bhusan ${ }^{\mathrm{c}}$ \\ ${ }^{a}$ Formerly CSIR-NPL, New Delhi (Present Address: 114 DayanandVihar, Delhi 110092), India \\ ${ }^{b}$ Formerly CSIR-NISTADS, New Delhi (Present Address: 1173 Sector 15, Panchkula 134 113, Haryana), India \\ ${ }^{c}$ Defence Scientific Information \& Documentation Centre, Delhi 11 054, India
}

\begin{abstract}
The paper maps quantum computing research on various publication and citation indicators, using data from Scopus database covering 10-year period 2007-16. Quantum computing research cumulated 4703 publications in 10 years, registered a slow 3.39\% growth per annum, and averaged 14.30 citations per paper during the period. Top 10 countries dominate the field with $93.15 \%$ global publications share. The USA accounted for the highest $29.98 \%$ during the period. Australia tops in relative citation index (2.0). International collaboration has been a major driver of research in the subject; $14.10 \%$ to $62.64 \%$ of national level output of top 10 countries appeared as international collaborative publications. Computer Science is one of the most popular areas of research in quantum computing research. The study identifies top 30 most productive organizations and authors, top 20 journals reporting quantum computing research, and 124 highly cited papers with $100+$ citations per paper.
\end{abstract}

Keywords:

Quantum computing;

Computer Science;

Global publications;

Scientometrics;

Bibliometrics.

Article History:

Received: $30 \quad$ May 2018

Accepted: 18 August 2018

\section{1- Introduction}

Quantum computing is a new genre of computing radically different from classical computing [1]. Classical computers use principles of 'classical' physics to store information in 'bits', each of which is always in one of the two states, either a 0 or a 1 (like as in an on/off switch). Quantum computers use effects of quantum physics to encode information in quantum bits, or qubits. A single qubit can store both a 0 and a 1 simultaneously, or any quantum superposition of these two qubit states. Computing in quantum computers is million times more powerful than in the classical computers. For example, a sequence of 4 bits/qubits can be in $2^{4}=16$ states (values). But a classical computer can pick only one of the values out of the total $2^{4}=16$ available at a time in its computing. Whereas a quantum computer processing 4 qubits will process all of these 16 states (numbers) and can do $2^{16}$ or 65,536 calculations in a single step and store computations exponentially [2,3]. A sort of parallelism in quantum computing is what makes quantum computers more powerful and faster than most powerful supercomputers of today [4].

Classical computers nowadays have reached the maximum limit of their computational power and speed of computation. Technologically, the transistors-the basic switching and memory unit of classical computers - have already reached their smallest ever physical size to atomic level. Further reduction in their physical size to sub-atomic level raises performance issues due to unstable nature of particles (qubits) at this level. At sub atomic levels, unstable particles stop obeying classical laws of physics, instead rules change and they (unstable particles) start obeying laws of quantum physics, exhibit a tunnelling effect which eventually disrupts the conventional binary computation process in a transistor. Given such limits to making improvements in building perfect transistors at sub-atomic level, scientists are forging ahead with new initiatives to pave the way for development of quantum computers that promise hope to provide faster

\footnotetext{
* CONTACT: Bmgupta1@ gmail.com

DOI: http://dx.doi.org/10.28991/esj-2018-01147

(C) This is an open access article under the CC-BY license (https://creativecommons.org/licenses/by/4.0/).
} 
and more powerful computers in future $[5,6]$.

Quantum computers are billed as fifth generation computers with major potential to solve so many different problems that are too complex or even impossible for today's computers. However, quantum computing technologies are still in their infancy stage and it will take years before such technologies are likely to come up for use on commercial scale. The ongoing developments in quantum computing technologies are mainly limited to developed world countries like USA, U.K., Canada, Japan, China, and Australia, [7- 9]. Quantum computing being a booming area of research, it would be worthwhile to map quantum computing research with a view to provide a quantitative and qualitative description of the subject under study.

\section{1-1- Literature Review}

Octamine provides a quantitative description of patenting activity in quantum computing research covering the period 1994-2014. According to this report, patenting activity has followed a growth in three cycles. Despite developments picking in 2013, breakthroughs in quantum computing technologies are still far way [10]. Scanlon, Tony, and Mishra [11] in their report analysed three areas of quantum computing patents: qubits, hardware, and applications of quantum computing. Projections are that by 2017, qubit technologies will see the most patent family publications, followed closely by quantum hardware patents, and applications of patents. The report maps emerging quantum companies, the academic world and their patenting activities [11]. No other studies are available as yet in the body of literature on bibliometric studies covering quantitative and qualitative description of quantum computing research per se. However, a number of bibliometric studies do exist on computer science research in general [8-9, 12-19] and its subfields such as artificial intelligence [18, 20], cloud computing [19-22], cryptography[21, 23], computational intelligence[22, 24], granular computing [23, 25], grid computing[24, 26], parallel computing [25, 27], pervasive and ubiquitous computing [26, 28], and software engineering [27, 29], etc. in particular.

\section{2- Objectives}

The study has been undertaken with the intent to map the global quantum computing research as indexed in Scopus international database during 2007-16. The study seeks to describe the subject in terms its growth rate, citation impact, international collaborative publications, research output by top 10 most productive countries; distribution of research by broad subject areas, the dynamics of research growth and decline across sub-areas of quantum computing over time; publication and citation profile of top 30 most productive research organizations and authors; main modes of communication, and the bibliometric profile of top 124 highly cited papers.

\section{3- Methodology}

The study retrieved and downloaded 10-year publications data on quantum computing research using Scopus database (http://www.scopus.com) covering the publication period 2007-16. For downloading data a search string was formulated. The term "quantum computing" was used as the keyword and tagged it to "Keyword" and "Source Title" search fields. To limit the global research output to publication period '2007-16', the search string formulated as stated above was conjoined with date range search field using Boolean Operator 'AND'. In the subsequent round of searching, the final search output was refined by using "subject area tag", "country tag", "source title tag", "journal title name" and "affiliation tag", in order to get data/information on the distribution of global publications output by subject, collaborating countries, author-wise, organization-wise and journal-wise, etc. For citation data, citations to publications were collected from date of publication till 13 November 2017.

(KEY (quantum computing) OR TITLE (quantum computing)) AND PUBYEAR > 2006 AND PUBYEAR < 2017

\section{4 -Analysis}

The 10-year quantum computing research across the world cumulated to 4703 publications during 2007-16, and it registered 3.39\% growth, up from 391 in 2007 to 509 publications in 2016. The 5-year global output in the subject registered $7.73 \%$ absolute growth, up from 2264 in 2007-11 to 2439 publications in 2012-16. The 10-year citation impact of quantum computing research averaged to 14.30 citations per publication (CPP) during 2007-16. The 5-year citation impact of research in the subject declined from 21.76 CPP during 2007-11 to 7.38 CPP during 2012-16 (Table 1, Figure $1)$. 
Table 1. Global Publications Output in Quantum Computing Research, 2007-16.

\begin{tabular}{cccc}
\hline \multirow{2}{*}{ Publication Period } & \multicolumn{3}{c}{ World } \\
\cline { 2 - 4 } & TP & TC & CPP \\
\hline 2007 & 391 & 13184 & 33.72 \\
2008 & 455 & 12820 & 28.18 \\
2009 & 464 & 9978 & 21.50 \\
2010 & 436 & 7004 & 16.06 \\
2011 & 518 & 6268 & 12.10 \\
2012 & 513 & 5980 & 11.66 \\
2013 & 449 & 4631 & 10.31 \\
2014 & 469 & 3626 & 7.73 \\
2015 & 499 & 2713 & 5.44 \\
2016 & 509 & 1052 & 2.07 \\
$2007-11$ & 2264 & 49254 & 21.76 \\
$2012-16$ & 2439 & 18002 & 7.38 \\
$2007-16$ & 4703 & 67256 & 14.30 \\
\hline
\end{tabular}

TP=Total Papers; TC=Total Citations; $\mathrm{CPP}=$ Citations Per Paper

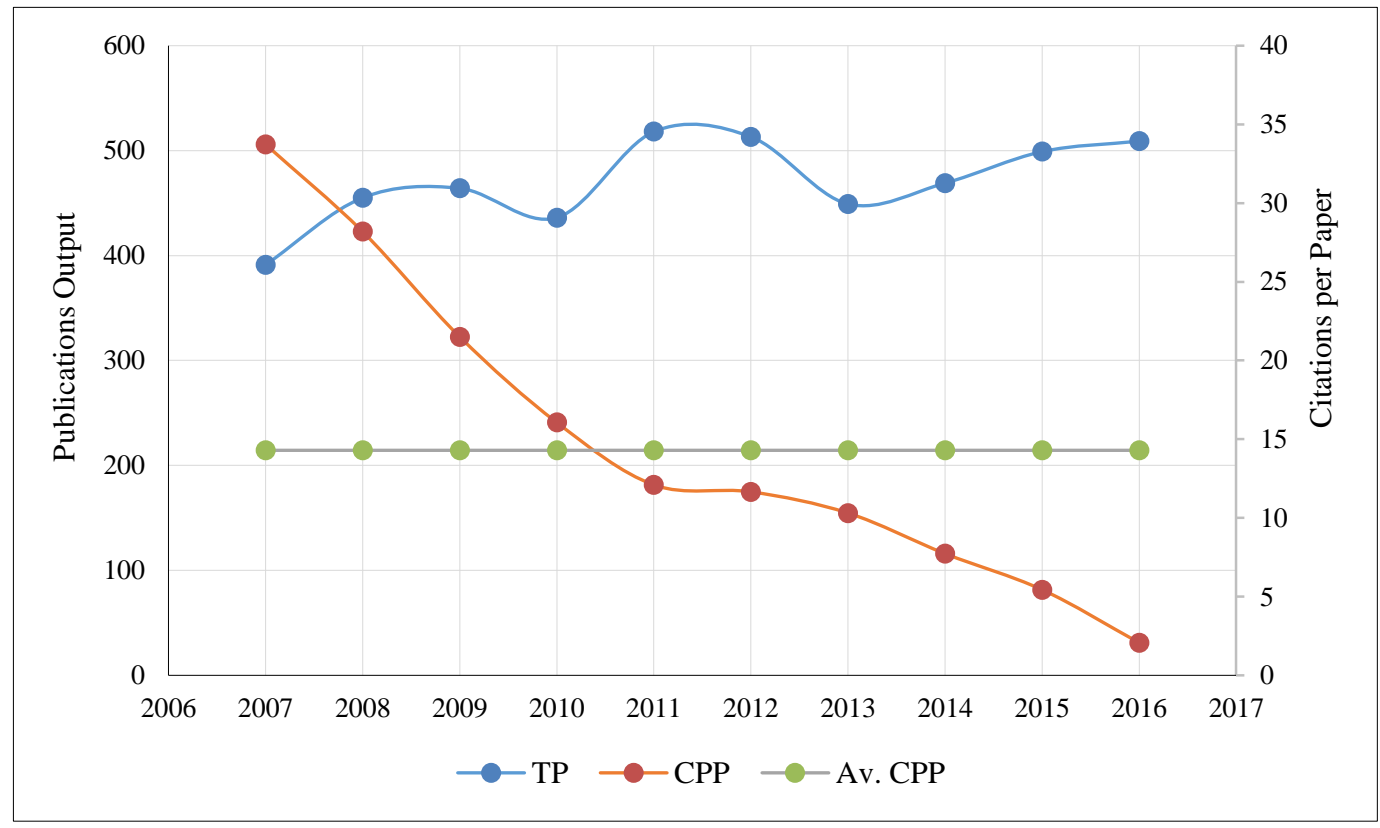

Figure 1. Publications and Citations Output in Quantum Computing Research Across the World.

Of the total global publications output, $53.65 \%$ (2523) appeared as articles, 38.19\% (1796) as conference papers, $3.38 \%$ (159) as reviews, $2.36 \%$ (111) as book chapters, $0.68 \%$ (32) as short surveys, $0.51 \%(24)$ as books, $0.43 \%(20)$ as editorials, $0.30 \%(14)$ as notes, $0.28 \%(13)$ as conference reviews and $0.23 \%(11)$ as articles in press.

\section{4-1- Top 10 Most Productive Countries in the World in Quantum Computing Research}

The global research output in quantum computing field originated from nearly 90 countries during 2007-16. The distribution of global publications output by participating countries is skewed. Of the 90 participating countries, 30 contributed 1-10 papers each, 29 countries 11-50 papers each, 11 countries 51-100 papers each, 10 countries 101-1000 papers each and 1 country 1001-5000 paper. 
Table 2. Global Publication Share of Top 10 Most Productive Countries in Quantum Computing Research during 2007-16.

\begin{tabular}{|c|c|c|c|c|c|c|c|c|c|c|c|c|}
\hline \multirow{2}{*}{$\begin{array}{c}S . N \\
o\end{array}$} & \multirow{2}{*}{$\begin{array}{l}\text { Name of the } \\
\text { Country }\end{array}$} & \multicolumn{3}{|c|}{ Total Papers } & \multicolumn{3}{|c|}{ \% Share of Global Papers } & TC & CPP & $\begin{array}{c}\mathbf{R C} \\
\mathbf{I}\end{array}$ & ICP & $\% \mathrm{ICP}$ \\
\hline & & 2007-11 & $2012-16$ & $2007-16$ & $2007-11$ & 2012-16 & $2007-16$ & \multicolumn{5}{|c|}{$2007-16$} \\
\hline 1 & USA & 703 & 707 & 1410 & 31.05 & 28.99 & 29.98 & 30266 & 21.47 & 1.50 & 441 & 31.28 \\
\hline 2 & China & 335 & 387 & 722 & 14.80 & 15.87 & 15.35 & 6674 & 9.24 & 0.65 & 187 & 25.90 \\
\hline 3 & U.K. & 160 & 249 & 409 & 7.07 & 10.21 & 8.70 & 9796 & 23.95 & 1.67 & 251 & 61.37 \\
\hline 4 & Germany & 172 & 192 & 364 & 7.60 & 7.87 & 7.74 & 10201 & 28.02 & 1.96 & 228 & 62.64 \\
\hline 5 & Japan & 159 & 159 & 318 & 7.02 & 6.52 & 6.76 & 4623 & 14.54 & 1.02 & 140 & 44.03 \\
\hline 6 & India & 84 & 221 & 305 & 3.71 & 9.06 & 6.49 & 3450 & 11.31 & 0.79 & 43 & 14.10 \\
\hline 7 & Canada & 150 & 136 & 286 & 6.63 & 5.58 & 6.08 & 7766 & 27.15 & 1.90 & 176 & 61.54 \\
\hline 8 & Australia & 110 & 113 & 223 & 4.86 & 4.63 & 4.74 & 6391 & 28.66 & 2.00 & 120 & 53.81 \\
\hline 9 & Italy & 99 & 80 & 179 & 4.37 & 3.28 & 3.81 & 3339 & 18.65 & 1.30 & 83 & 46.37 \\
\hline 10 & France & 75 & 90 & 165 & 3.31 & 3.69 & 3.51 & 3150 & 19.09 & 1.34 & 96 & 58.18 \\
\hline \multicolumn{2}{|c|}{ Total of 10 countries } & 2047 & 2334 & 4381 & 90.42 & 95.69 & 93.15 & 85656 & 19.55 & 1.37 & 1765 & 40.29 \\
\hline \multicolumn{2}{|c|}{ World Total } & 2264 & 2439 & 4703 & & & & 67256 & 14.30 & & & \\
\hline \multicolumn{2}{|c|}{$\begin{array}{c}\text { Share of } 10 \text { Countries in } \\
\text { World Total }\end{array}$} & 90.42 & 95.69 & 93.15 & & & & & & & & \\
\hline
\end{tabular}

* TC=Total Citations; $\mathrm{CPP}=$ Citations Per Paper; ICP=International Collaborative Papers; RCI=Relative Citation Index

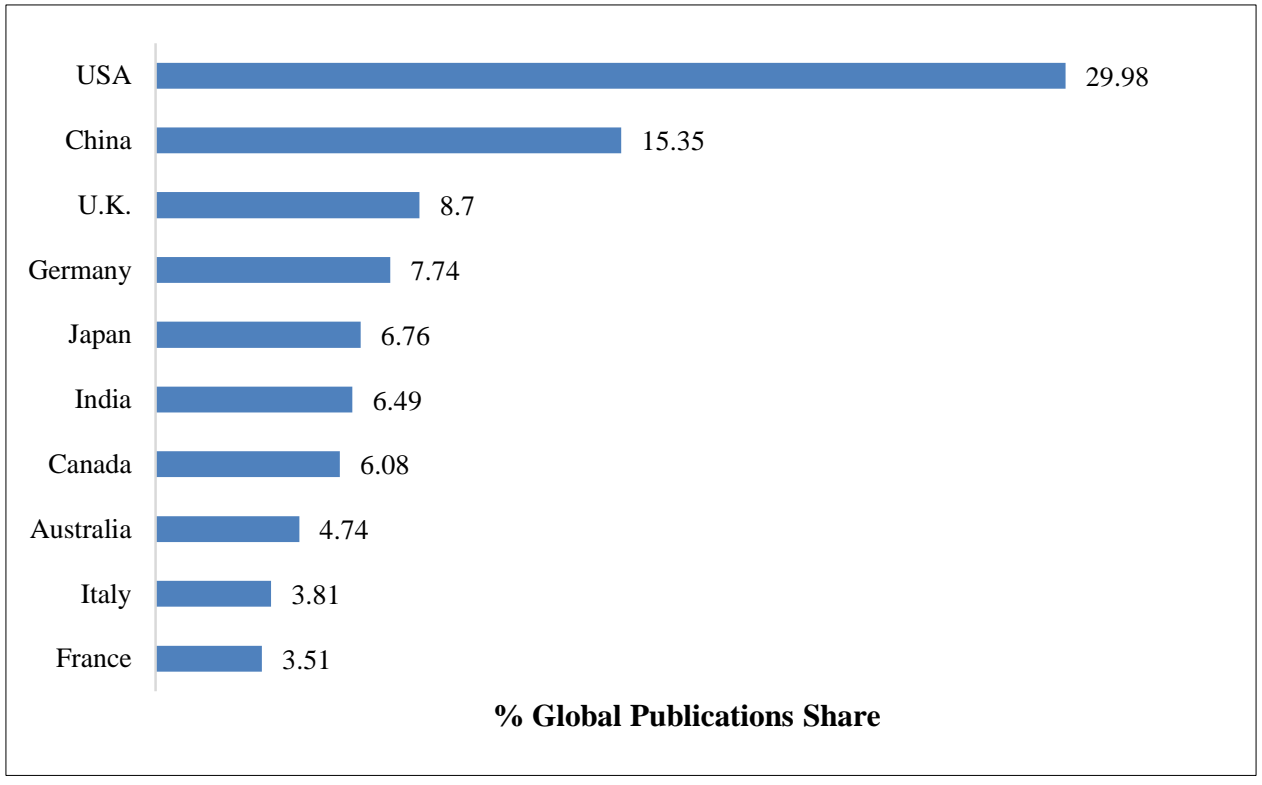

Figure 2. Global Publications Share of Top Countries in Quantum Computing Research during 2007-2016.

Top 10 countries dominated quantum computing research with $93.15 \%$ global publication share during the period (Table 2). Individually their global publication share varied from $3.51 \%$ to $29.98 \%$. The USA contributed the highest $29.98 \%$ share, followed by China (15.35\%), U.K. (8.70\%), etc. Italy and France positioned at bottom end of the tally contributed $3.51 \%$ to $3.81 \%$ global publications share.

\section{4-2- International Collaboration}

The international collaborative share (ICP) of top 10 most productive countries in quantum computing research varied from $14.10 \%$ to $62.64 \%$ of their national publications output, with an average share of $38.26 \%$. The highest ICP share was reported by Germany, Canada and U.K. (62.64\%, 61.54\% and 61.37\%), followed by France and Australia (58.18\% and 53.81\%), Italy and Japan (46.37\% and 44.03\%), USA (31.28\%), China (25.90\%) and India (14.10\%) in 10 years during 2007-16. 


\section{4-3- Distribution of Research Output by Sub-Fields}

The global publication output in quantum computing research is distributed across nine sub-fields as identified in Scopus database classification. Computer science is the most popular area of research in quantum research accounting for the highest global publications share (44.89\%), followed by physics and astronomy (39.55\%), engineering (29.0\%), mathematics $(21.90 \%)$, materials science $(14.18 \%)$, chemistry $(9.97 \%)$ and other sub-field from $2.66 \%$ to $5.44 \%$ during 2007-16.

Medicine among 9 sub-fields, registered the highest citations per paper of 55.69, followed chemical engine (30.34), chemistry (22.80), biochemistry, genetics and molecular biology (20.36), physics and astronomy (16.92), materials science (14.54), engineering (14.54), mathematics (6.84) and computer science (6.47) during 2007-16 (Table 3).

Table 3. Subject-Wise Breakup of Global Publications in Quantum Computing Research during 2007-16.

\begin{tabular}{|c|c|c|c|c|c|c|c|c|c|}
\hline \multirow{2}{*}{ S.No } & \multirow{2}{*}{ Subject* } & \multicolumn{3}{|c|}{ Number of Papers (TP) } & \multicolumn{2}{|c|}{ Activity Index } & \multirow{2}{*}{$\begin{array}{c}\mathrm{TC} \\
2007-16\end{array}$} & \multirow{2}{*}{$\begin{array}{c}\text { CPP } \\
2007-16\end{array}$} & \multirow{2}{*}{$\frac{\% \mathrm{TP}}{2007-16}$} \\
\hline & & 2007-11 & 2012-16 & $2007-16$ & 2007-11 & 2012-16 & & & \\
\hline 1 & Computer Science & 995 & 1116 & 2111 & 97.91 & 101.94 & 13658 & 6.47 & 44.89 \\
\hline 2 & Physics \& Astronomy & 872 & 988 & 1860 & 97.39 & 102.43 & 31479 & 16.92 & 39.55 \\
\hline 3 & Engineering & 645 & 719 & 1364 & 98.23 & 101.64 & 11309 & 8.29 & 29.00 \\
\hline 4 & Mathematics & 488 & 542 & 1030 & 98.42 & 101.47 & 7045 & 6.84 & 21.90 \\
\hline 5 & Materials Science & 283 & 384 & 667 & 88.14 & 111.01 & 9701 & 14.54 & 14.18 \\
\hline 6 & Chemistry & 241 & 228 & 469 & 106.74 & 93.74 & 10692 & 22.80 & 9.97 \\
\hline 7 & $\begin{array}{c}\text { Biochemistry, Genetics \& } \\
\text { Molecular Biology }\end{array}$ & 135 & 121 & 256 & 109.54 & 91.14 & 5213 & 20.36 & 5.44 \\
\hline 8 & Chemical Engineering & 81 & 84 & 165 & 101.98 & 98.17 & 5006 & 30.34 & 3.51 \\
\hline 9 & Medicine & 52 & 73 & 125 & 86.42 & 112.61 & 6961 & 55.69 & 2.66 \\
\hline & World Output & 2264 & 2439 & 4703 & & & & & \\
\hline \multicolumn{10}{|c|}{ There is overlapping of literature covered under various subjects } \\
\hline \multicolumn{10}{|c|}{$\mathrm{TP}=$ Total Papers; $\mathrm{TC}=$ Total Citations $\mathrm{CPP}=$ Citations Per Paper } \\
\hline
\end{tabular}

\section{4-4- Profile of Top 30 Global Organizations}

A total of 1642 organizations published 4703 publications in quantum computing research with an average of 2.86 publications per organization. The distribution of publication output across participating organizations is highly skewed. Of the 1642 organizations, 1301 contributed 1-5 papers each, 190 organizations 6-10 papers each, 134 organizations 11 50 papers each, 13 organizations 51-100 papers each and 4 organizations 101-160 papers each.

The publication productivity of top 30 most productive organizations in quantum computing research across the world varied from 30 to 102 publications. Together they accounted for $28.53 \%$ (1342) publication share and $65.36 \%$ (43958) citation share of total publications output in 10 years during 2007-16. that:

The scientometric profile of these top 30 organizations is presented in Table 4. On further analysis, it was observed

- Ten of top 30 organizations registered publications output above the group average of 44.73: University of Oxford, U.K., University of Waterloo, Canada, Massachusetts Institute of Tech., USA, National University of Singapore, etc. during 2007-16.

- Eleven organizations registered citation impact above the group average of 32.76 citations per publication: Stanford University, USA (103.31), Princeton University, USA (98.71), University of California, Berkeley, USA (66.84), University of Queensland, Australia (66.71), Osterreichische Akademie Der Wissenschaften, Austria (52.94), etc. during 2007-16.

- Fifteen organizations contributed international collaborative publications above the group average of share of 51.86\%: National University of Singapore (88.89\%), Perimeter Institute of Theoretical Physics, Waterloo, Canada (80.56\%), University of Bristol, U.K.(70.97\%), University of Queensland, Australia (66.67\%), University of Waterloo, Canada (65.66\%), etc. during 2007-16; and

- Eleven organizations registered their relative citation index above the group average (2.29) of all organizations: Stanford University, USA (7.22), Princeton University, USA (6.9), University of Queensland, Australia (4.67), University of California, Berkeley, USA (4.67), Osterreichische Akademie Der Wissenschaften, Austria (3.7), etc. during 2007-16. 
Table 4. Scientometric Profile of Top 30 Most Productive Global Organizations on Quantum Computing Research during 2007-16.

\begin{tabular}{|c|c|c|c|c|c|c|c|c|}
\hline S.No & Name of the Organization & TP & TC & CPP & HI & ICP & $\% \mathrm{ICP}$ & RCI \\
\hline 1 & University of Oxford, U.K. & 102 & 2928 & 28.71 & 25 & 62 & 60.78 & 2.01 \\
\hline 2 & University of Waterloo, Canada & 99 & 2915 & 29.44 & 24 & 65 & 65.66 & 2.06 \\
\hline 3 & Massachusetts Institute of Techy, USA & 63 & 1515 & 24.05 & 17 & 30 & 47.62 & 1.68 \\
\hline 4 & National University of Singapore & 63 & 1175 & 18.65 & 19 & 56 & 88.89 & 1.30 \\
\hline 5 & CNRS Centre National de la Recherché Scientifique, France & 61 & 765 & 12.54 & 13 & 33 & 54.10 & 0.88 \\
\hline 6 & Harvard University, USA & 51 & 1992 & 39.06 & 17 & 30 & 58.82 & 2.73 \\
\hline 7 & Tsinghua University, China & 51 & 890 & 17.45 & 17 & 21 & 41.18 & 1.22 \\
\hline 8 & $\begin{array}{l}\text { Research Organization of Information \& Systems National } \\
\text { Institute of Informatics, Japan }\end{array}$ & 51 & 1741 & 34.14 & 13 & 31 & 60.78 & 2.39 \\
\hline 9 & University of California, Berkeley, USA & 49 & 3275 & 66.84 & 16 & 25 & 51.02 & 4.67 \\
\hline 10 & University of Tokyo, Japan & 45 & 589 & 13.09 & 12 & 15 & 33.33 & 0.92 \\
\hline 11 & Ministry of Education of China & 42 & 362 & 8.62 & 11 & 8 & 19.05 & 0.60 \\
\hline 12 & National Institute of Standards \& Technology, USA & 42 & 1569 & 37.36 & 16 & 16 & 38.10 & 2.61 \\
\hline 13 & University of Queensland, Australia & 42 & 2802 & 66.71 & 18 & 28 & 66.67 & 4.67 \\
\hline 14 & Stanford University, USA & 39 & 4029 & 103.31 & 19 & 25 & 64.10 & 7.22 \\
\hline 15 & Japan Science \& Technology Agency & 39 & 503 & 12.90 & 12 & 9 & 23.08 & 0.90 \\
\hline 16 & University of Sydney, Australia & 38 & 757 & 19.92 & 13 & 20 & 52.63 & 1.39 \\
\hline 17 & University of Melbourne, Australia & 38 & 1147 & 30.18 & 15 & 21 & 55.26 & 2.11 \\
\hline 18 & Perimeter Institute of Theoretical Physics, Waterloo, Canada & 36 & 1083 & 30.08 & 15 & 29 & 80.56 & 2.10 \\
\hline 19 & California Institute of Technology, Pasadenia, USA & 36 & 1253 & 34.81 & 16 & 18 & 50.00 & 2.43 \\
\hline 20 & University of Science \& Techn of China & 35 & 794 & 22.69 & 15 & 20 & 57.14 & 1.59 \\
\hline 21 & Osterreichische Akademie Der Wissenschaften, Austria & 34 & 1800 & 52.94 & 19 & 22 & 64.71 & 3.70 \\
\hline 22 & University of Innsbruck, Austria & 33 & 1342 & 40.67 & 15 & 21 & 63.64 & 2.84 \\
\hline 23 & University of Maryland, USA & 33 & 920 & 27.88 & 11 & 7 & 21.21 & 1.95 \\
\hline 24 & Nippon Telegraph \& Telephone Corporation, Japan & 33 & 367 & 11.12 & 8 & 7 & 21.21 & 0.78 \\
\hline 25 & University of South Florida Tampa, USA & 32 & 978 & 30.56 & 14 & 5 & 15.63 & 2.14 \\
\hline 26 & University of California, Santa Barbara, USA & 32 & 1401 & 43.78 & 14 & 14 & 43.75 & 3.06 \\
\hline 27 & Princeton University, USA & 31 & 3060 & 98.71 & 14 & 12 & 38.71 & 6.90 \\
\hline 28 & Sandia National Laboratories, New, Mexico, USA & 31 & 283 & 9.13 & 7 & 9 & 29.03 & 0.64 \\
\hline 29 & University of Bristol, U.K. & 31 & 980 & 31.61 & 13 & 22 & 70.97 & 2.21 \\
\hline \multirow[t]{4}{*}{30} & Purdue University, USA & 30 & 743 & 24.77 & 9 & 15 & 50.00 & 1.73 \\
\hline & Total of 30 organizations & 1342 & 43958 & 32.76 & 14.9 & 696 & 51.86 & 2.29 \\
\hline & Total of World & 4703 & 67256 & 14.30 & & & & \\
\hline & Share of top 30 organizations in World total output & 28.53 & 65.36 & & & & & \\
\hline
\end{tabular}

TP=Total Papers; TC=Total Citations; CPP=Citations Per Paper; HI=h-index; ICP=International Collaborative Papers; RCI=Relative Citation Index

\section{4-5- Profile of Top 30 Most Productive Authors}

A total of 2395 authors across the world participated in quantum computing research, of which 2036 published 1-5 papers each, 218 authors 6-10 papers each, 109 authors 11-20 papers each and 32 authors 21-90 papers each. The research productivity of top 30 authors in quantum computing research varied from 12 to 26 publications. Together they contributed $9.84 \%$ (463) global publication share and 21.91\% (14735) citation share of total research output in 10 years during 2007-16. The scientometric profile of these 30 authors is presented in Table 5.

On further analysis, it was observed that:

- Nine of top 30 authors registered publications output above the group average of 15.43: N. Ranganathan (26 papers), M. Haghparast (25 papers), H. Thapliyal (24 papers), S. Bhatttacharyya (20 papers), E. Kashefi (19 papers), etc. during 2007-16.

- Ten authors registered citation impact above the group average of 31.83 citations per publication: T.C. Ralph (101.93), P. Kok (96.25), A. Aspuru-Guzik (92.92), W.J. Munro (77.67), K. Nemoto (72.83), etc. during 200716. 
- Seventeen authors contributed international collaborative publications above the group average share of $40.60 \%$ of all authors: S.T. Flammia (91.67\%), E. Kashefi (78.95\%), A.G. Fowler (76.47\%), A. Ambainis (75.0\%), D. Qiu (73.33\%), etc. during 2007-16; and.

- Ten authors registered the relative citation index above the group average (2.23) of all authors: T.C. Ralph (7.13), P.Kok (6.73), A. Aspuru-Guzik (6.5), W.J. Munro (5.43), K. Nemoto (5.09), etc. during 2007-16.

Table 5. Scientometric Profile of Top 30 Most Productive Authors in Quantum Computing Research during 2007-16.

\begin{tabular}{|c|c|c|c|c|c|c|c|c|c|}
\hline \multirow{2}{*}{ S.No } & Name of the & \multirow{2}{*}{ Affiliation of the Author } & \multirow{2}{*}{$\mathbf{T P}$} & \multirow{2}{*}{ TC } & \multirow{2}{*}{ CPP } & \multirow{2}{*}{ HI } & \multirow{2}{*}{ ICP } & \multirow{2}{*}{$\% \mathrm{ICP}$} & \multirow{2}{*}{ RCI } \\
\hline & Author & & & & & & & & \\
\hline 1 & N. Ranganathan & University of Southern Florida, USA & 26 & 411 & 15.81 & 12 & 0 & 0.00 & 1.11 \\
\hline 2 & M. Haghparast & Islamic Azad University, Tehran, Iran & 25 & 323 & 12.92 & 7 & 0 & 0.00 & 0.90 \\
\hline 3 & H. Thapliyal & University of Southern Florida, USA & 24 & 358 & 14.92 & 12 & 3 & 12.50 & 1.04 \\
\hline 4 & $\begin{array}{c}\text { S. } \\
\text { Bhatttacharyya }\end{array}$ & RCC Institute of Information Technology, Kolkata & 20 & 122 & 6.10 & 6 & 2 & 10.00 & 0.43 \\
\hline 5 & E. Kashefi & $\begin{array}{l}\text { Institute of Quantum Optics \& Quantum Information } \\
\text { Sciences, Austrian Academy of Sciences, Vienna, Austria }\end{array}$ & 19 & 452 & 23.79 & 8 & 15 & 78.95 & 1.66 \\
\hline 6 & W.J. Munro & Hewlett Packard Laboratories, Bristol, U.K. & 18 & 1398 & 77.67 & 11 & 13 & 72.22 & 5.43 \\
\hline 7 & K. Nemoto & National Institute of Informatics, Tokyo, Japan & 18 & 1311 & 72.83 & 18 & 10 & 55.56 & 5.09 \\
\hline 8 & A.G. Fowler & University of Melbourne, Australia & 17 & 962 & 56.59 & 11 & 13 & 76.47 & 3.96 \\
\hline 9 & R. Wille & University of Bremen, Germany & 17 & 448 & 26.35 & 8 & 8 & 47.06 & 1.84 \\
\hline 10 & A.N. Al-Rabadi & University of Jordan, Amman, Jordan & 15 & 25 & 1.67 & 3 & 6 & 40.00 & 0.12 \\
\hline 11 & R. Blatt & Osterreichische Akademe der Wissenschaften, Austria & 15 & 1018 & 67.87 & 10 & 6 & 40.00 & 4.75 \\
\hline 12 & T. Morimae & Gunma University, Japan & 15 & 166 & 11.07 & 8 & 10 & 66.67 & 0.77 \\
\hline 13 & D. Qiu & Zhongshan University, China & 15 & 107 & 7.13 & 7 & 11 & 73.33 & 0.50 \\
\hline 14 & .S. Aarsonson & Massachusetts Institute of Technology, USA & 14 & 176 & 12.57 & 7 & 4 & 28.57 & 0.88 \\
\hline 15 & S.C. Benjamin & University of Oxford, U.K. & 14 & 253 & 18.07 & 8 & 9 & 64.29 & 1.26 \\
\hline 16 & L.C. Hollenberg & University of Melbourne, Australia & 15 & 317 & 21.13 & 6 & 7 & 46.67 & 1.48 \\
\hline 17 & A.Lagana & University of Perugia, Italy & 14 & 121 & 8.64 & 6 & 5 & 35.71 & 0.60 \\
\hline 18 & T.C. Ralph & University of Queensland, Australia & 14 & 1427 & 101.93 & 9 & 7 & 50.00 & 7.13 \\
\hline 19 & H.E. Brandt & U.S. Army Research Lab, Adelephi, MD, USA & 13 & 21 & 1.62 & 3 & 0 & 0.00 & 0.11 \\
\hline 20 & R. Drechsler & University of Bremen, Germany & 13 & 338 & 26.00 & 7 & 5 & 38.46 & 1.82 \\
\hline 21 & O.Pfister & University of Virginia, USA & 13 & 407 & 31.31 & 5 & 9 & 69.23 & 2.19 \\
\hline 22 & M. Sedighi & Amirkabir University of Technology, Tehran, Iran & 13 & 74 & 5.69 & 6 & 0 & 0.00 & 0.40 \\
\hline 23 & A.Ambainis & University of Waterloo, Canada & 12 & 242 & 20.17 & 7 & 9 & 75.00 & 1.41 \\
\hline 24 & A.Aspuru-Guzik & Harvard University, USA & 12 & 1115 & 92.92 & 9 & 5 & 41.67 & 6.50 \\
\hline 25 & S.T.Flammia & Perimeter Institute of Theoretical Physics, Waterloo, Canada & 12 & 504 & 42.00 & 7 & 11 & 91.67 & 2.94 \\
\hline 26 & G.Klimeck & Purdue University, USA & 12 & 122 & 10.17 & 6 & 6 & 50.00 & 0.71 \\
\hline 27 & P.Kok & University of Sheffield, U.K. & 12 & 1155 & 96.25 & 4 & 1 & 8.33 & 6.73 \\
\hline 28 & S.J. Lomonaco & University of Maryland, Baltimore, USA & 12 & 514 & 42.83 & 9 & 6 & 50.00 & 3.00 \\
\hline 29 & K. Navi & Shahid Beheshti University, Tehran, Iran & 12 & 334 & 27.83 & 6 & 1 & 8.33 & 1.95 \\
\hline 30 & K. Molmer & University of Aarhus, Denmark & 12 & 514 & 42.83 & 9 & 6 & 50.00 & 3.00 \\
\hline & & Total of 30authors & 463 & 14735 & 31.83 & 7.83 & 188 & 40.60 & 2.23 \\
\hline & & Total of World & 4703 & 67256 & 14.30 & & & & \\
\hline \multicolumn{3}{|c|}{ Share of top 30 authors in World total output } & 9.84 & 21.91 & & & & & \\
\hline
\end{tabular}

TP=Total Papers; TC=Total Citations; CPP=Citations Per Paper; HI=h-index; ICP=International Collaborative Papers; RCI=Relative Citation Index

\section{4-6- Medium of Research Communication}

Of the global research output in quantum computing field, 60.05\% (2824) appeared in journals, $29.11 \%$ (1369) in conference proceedings, $7.08 \%$ (333) in book series, $2.83 \%$ (133) as books and $0.77 \%$ (36) as trade publications and $0.67 \%(58)$ as books. Of The top 20 most productive journals which published 21 to 198 papers each, accounted for $33.44 \%$ share (947 papers) of total papers in journal medium in 10 years during 2007-16. The top most productive journals include: Journal of Physical Review A (with 198 papers), followed by Physical Review Letters (139 papers), 
New Journal of Physics (82 papers), Quantum Information Processing (60 papers), Computer Physics Communication (49 papers), etc. during 2007-16 (Table 6).

Table 6. Top 20 Most Productive Journals in Quantum Computing Research during 2007-16.

\begin{tabular}{|c|c|c|c|c|}
\hline \multirow{2}{*}{ S.No } & \multirow{2}{*}{ Name of the Journal } & \multicolumn{3}{|c|}{ Number of Papers } \\
\hline & & $2007-11$ & 2012-16 & 2007-16 \\
\hline 1 & Physical Review A & 97 & 101 & 198 \\
\hline 2 & Physical Review Letters & 57 & 82 & 139 \\
\hline 3 & New Journal of Physics & 30 & 52 & 82 \\
\hline 4 & Quantum Information Processing & 13 & 47 & 60 \\
\hline 5 & Computer Physics Communication & 27 & 22 & 49 \\
\hline 6 & Quantum Information \& Communication & 24 & 19 & 43 \\
\hline 7 & Journal of Physics Conference Series & 14 & 22 & 36 \\
\hline 8 & Nature & 26 & 10 & 36 \\
\hline 9 & Nature Computing & 18 & 16 & 34 \\
\hline 10 & Journal of Chemical Physics & 18 & 12 & 30 \\
\hline 11 & Applied Physics Letters & 9 & 19 & 28 \\
\hline 12 & Theoretical Computer Science & 17 & 10 & 27 \\
\hline 13 & IEEE Transaction on Applied Superconductivity & 13 & 13 & 26 \\
\hline 14 & International Journal of Quantum Information & 18 & 8 & 26 \\
\hline 15 & Proceeding of National Academy of Sciences of & 16 & 8 & 24 \\
\hline 16 & Journal of Computational Chemistry & 9 & 13 & 22 \\
\hline 17 & Nanotech & 8 & 14 & 22 \\
\hline 18 & PLOS One & 3 & 19 & 22 \\
\hline 19 & Science & 15 & 7 & 22 \\
\hline \multirow[t]{3}{*}{20} & Journal of Molecular Modelling & 16 & 5 & 21 \\
\hline & Total of 20 journals & 448 & 499 & 947 \\
\hline & Total global journal output & 1345 & 1487 & 2832 \\
\hline \multicolumn{2}{|r|}{ Share of top 20 journals in global journal output } & 33.31 & 33.56 & 33.44 \\
\hline
\end{tabular}

\section{4-7- Highly Cited Papers}

For this study, all such papers that received 100 or 100 plus citations per paper in 10 years since their publication during 2007-16 have been acknowledged as highly cited papers in quantum computing field. A total of 124 papers were identified as highly cited papers, which accounted for $2.64 \%$ share of the total world output (4703 papers) in the subject during the period. The distribution of highly cited papers across discrete citations frequencies is highly skewed.

- Of the 124 highly cited papers, 83 were in citation range 101-200 citations per paper, 19 in the range 201-300 citations per paper, 9 in the range 301-500 citations per paper, 7 in the range 501-900 citations per paper, 5 in the range 1001-2000 citations per paper and 1 lone paper cumulated 2705 citations since its publication during 2007 16.

- These 124 highly cited papers cumulated a total of 33046 citations, with an average of 266.50 citations per paper.

- Of the 124 highly cited papers, 42 were contributed by such individual organizations who pursued research in their standalone capacity (non-collaborative) and 82 by such other collaborating organizations who pursued research as a group of two or more organizations per paper (25 national collaborative and 57 as international collaborative organizations).

- Amongst highly cited papers, research participation by a country was the largest from USA (with 56 papers), followed by Canada (22 papers), Germany (18 papers), Australia (15 papers), U.K. (14 papers), China (11 papers), Italy, Spain and France ( 7 papers each), Japan and Netherland (5 papers), Poland and Israel (4 papers each, Belgium, Denmark, Taiwan and Switzerland (3 papers each), India , Singapore and South Korea (2 papers), Brazil, Russia Federation and Sweden (1 paper each).

- The 124 highly cited papers involved the participation of 720 authors from 379 organizations.

- The research organizations accounting for the largest number of highly cited papers include: University of Waterloo, Canada (9 papers), National Institute of Standards and Technology, USA and Stanford University, USA 
(6 papers each), University of California, Santa Barbara, USA, Princeton University, USA, Harvard University, USA, University of Berkeley, USA, Osterreichische Akademie Der Wissenshaften, Austria and Perimeter Institute of Theoretical Physics, Waterloo, Canada (5 papers each), University of Oxford, U.K. AND university OF Melbourne, Australia (4 papers each), MIT, USA, Tsinghua University, China, University of Innsbruck, Austria and University of Maryland, USA (3 papers), etc.

- The authors accounting for the largest number of highly cited papers include: A.G.Fowler (4 papers), T.C. Ralph, A. Aspuru-Guzik and S.T. Flammia (3 papers each), E. Kashefi, O. Pfister, J.W. Pan, N.C. Menicucci and R. Blatt, etc. (2 papers each).

- Of the 124 highly cited papers, 105 were published as articles, 14 as review papers and 5 as conference papers.

- These 124 highly cited papers were published across 50 national and international journals. Twenty one (21) papers appeared in Physical Review Letters, 13 papers in Nature, 8 papers in Science, 6 papers in Nature Photonics, 5 papers each in Journal of American Chemical Society and Physical Review A, 4 papers each in Nature Communications, Proceedings of NAS of USA and Review of Modern Physics, 2 papers each in Chemical Society Reviews, IEEE Transaction on Systems, Man \& Cybernetics. Part B, IEEE Transactions on Power Systems, Molecular Physics, Nature Physics, New Journal of Physics and SIAM Journal of Computing and 1 paper each in 34 other journals.

\section{5- Conclusion}

The present study provides a quantitative and qualitative description of quantum computing research across the world during 2007-16. The study sourced data for the study from the Scopus, an international multi-disciplinary database. Quantum computing research across the world cumulated a total of 4703 publications, and registered a slow 3.39\% growth during the period. The top 10 countries that dominate the world of quantum computing research include the USA, China, UK, Germany, Japan, India, Canada, Australia, Italy and France, with 93.15\% global publications share. Quantum computing research averaged a high citation impact of 14.30 citations per paper on a 10 -year citation window. Six of the top 10 most productive countries scored relative citation index above the group average of 1.33. Computer science is one of the most popular areas of study in quantum computing research, accounting for the highest subject share (44.89\%), followed by physics \& astronomy (39.55\%), and others. The top 30 most productive organizations in quantum computing research are mainly from developed world countries, like the USA (12), Japan (4), China (3), Australia (3), the U.K. (2), Austria (2), Canada (2), and France (1). Their citation impact averaged between 34.14 and $103.31 \mathrm{CPP}$ on a 10 -year window. Over $60 \%$ of global publications share in the subject appeared in journal literature, with top 20 journals accounting for $33.44 \%$ share of total output covered by journal medium.

Quantum computing research till recently 2016 has been intensely strong on quality, but not as much on quantity of research. That its body of literature is still too small and its growth too slow, alludes to a view that quantum computing research is still very much in its early stages of development. Even as quantum computing research efforts are global in scope, but leadership in the subject still rests with the USA. Of the top 30 research institutes in the subject across the world, as many as 12 are located in the USA, and the rest distributed in single digits across eight countries like the U.K, China, Singapore, Canada, Japan, Australia, Austria, and France.

\section{6- References}

[1] Bone, Simon, and Matias Castro. "A brief history of quantum computing." Imperial College in London, http://www. doc. ic. ac. $\mathrm{uk} / \sim$ nd/surprise_97/journa 1/vol4/spb3 (1997).

[2] Job One for Quantum Computers: Boost Artificial Intelligence. Quantum Magazine. https://www.quantamagazine.org/job-onefor-quantum-computers-boost-artificial-intelligence-20180129.

[3] What is Quantum Computing? (2013) the Economist. https://www.economist.com/blogs/economist-explains/2013/05/economistexplains- 10 .

[4] Deutsch, David. "Quantum computation." Physics World 5, no. 6 (1992): 57, doi:10.1088/2058-7058/5/6/38.

[5] Marr, Burmard. What is quantum computing, (2017). https://www.forbes.com/sites/bernardmarr/2017/07/04/what-is-quantumcomputing-a-super-easy-explanation-for-anyone.

[6] Woodford, Chris (2018). Quantum computing. http://www.explainthatstuff.com/quantum-computing.html.

[7] Roell, Jason. The Need, Promise, and Reality of Quantum Computing. https://towardsdatascience.com/the-need-promise-andreality-of-quantum-computing-4264ce15c6c0.

[8] Science Buff. Why Do We Need Quantum Computing? https://www.clerro.com/guide/485/why-do-we-need-quantumcomputing. 
[9] Introduction to Quantum Computing. Art of Engineer. https://artofengineer.com/articles/technology/introduction-to-quantumcomputing.

[10] Octimine technologies GmbH. Patents of Quantum Computing Companies.: Patent Reports. 2017. https://www.octimine.com/patents-of-quantum-computing-companies.

[11] Scanlon, Bryan, Tony, Anthony and Mishra, Gargi. Practical quantum computing: A patent landscape report. Patinfornmatics. LLC. 2017. http:/pateninformatics.com/quantum-computing-report.

[12] Gupta, B. M., Avinash Kshitij, and Charu Verma. "Mapping of Indian Computer Science Research Output, 1999-2008." Scientometrics 86, no. 2 (July 16, 2010): 261-283. Doi: 10.1007/s11192-010-0272-y.

[13] Gupta, B.M., Avinash Kshitij, and Yoginder Singh. "Indian Computer Science Research Output during 1999-2008: Qualitative Analysis." DESIDOC Journal of Library \& Information Technology 30, no. 6 (November 1, 2010): 39-54. doi:10.14429/djlit.30.680.

[14] Gupta, B.M., and S.M. Dhawan. "Highly Cited Publications Output by India in Computer Science 1996-15: A Scientometric Assessment.” Journal of Scientometric Research 6, no. 2 (October 3, 2017): 74-85. doi:10.5530/jscires.6.2.13.

[15] Bakri, Aryati, and Peter Willett. “Computer Science Research in Malaysia: a Bibliometric Analysis.” Edited by Peter Willett. Aslib Proceedings 63, no. 2/3 (March 22, 2011): 321-335. Doi: 10.1108/00012531111135727.

[16] Godoy, Daniela, Alejandro Zunino, and Cristian Mateos. "Publication Practices in the Argentinian Computer Science Community: a Bibliometric Perspective.” Scientometrics 102, no. 2 (October 15, 2014): 1795-1814. Doi: 10.1007/s11192-0141450-0.

[17] Adeniran, O.R. "Bibliometrics of Computer Science Literature in Nigeria.” International Library Review 20, no. 3 (July 1988): 347-359. doi:10.1016/0020-7837(88)90007-6.

[18] Uddin, Ashraf, Vivek Kumar Singh, David Pinto, and Ivan Olmos. "Scientometric Mapping of Computer Science Research in Mexico.” Scientometrics 105, no. 1 (August 13, 2015): 97-114. Doi: 10.1007/s11192-015-1654-y.

[19] Uddin, Ashraf, and Vivek Kumar Singh. "Mapping the Computer Science Research in SAARC Countries.” IETE Technical Review 31, no. 4 (July 4, 2014): 287-296. doi:10.1080/02564602.2014.947527.

[20] Niu, J., Tang, W., Xu, F., Zhou, X and Song, Y. Global research on artificial intelligence from 1990-2014: Spatially-explict bibiometric analysis. International Journal of Geo-Information 2016, 5, 66; doi: 10.3390/ijgi5050066.

[21] Gupta, B.M., Neeraj Kumar Singh, and Ritu Gupta. "International Cloud Computing Literature: A Scientometric Analysis for 2004-13.” Information Studies 21, no. 2and3 (2015): 11. doi:10.5958/0976-1934.2015.00010.5.

[22] Gupta, B. M., and Ritu Gupta. "Indian Cloud Computing Research: A Scientometric Assessment of Publications Output During 2004-13.” SRELS Journal of Information Management 52, no. 5 (October 1, 2015): 315. doi:10.17821/srels/2015/v52i5/79733.

[23]Baskaran, C. Scientometric analysis of cryptography research output. SRELS Journal of Information Management August 2013, 50(4), 413-421.

[24] Eck, Nees Jan Van. Bibliometric mapping of computational intelligence field. International Journal of Uncertainity, Fuzziness \& Knowledge-based Systems October 2007, 15(5). http://doi.org/10.1142/50218488507004911.

[25] Yao, JingTao. "Recent Developments in Granular Computing: A Bibliometrics Study.” 2008 IEEE International Conference on Granular Computing (August 2008). doi:10.1109/grc.2008.4664798.

[26] Santha Kumar, R. Grid computing research in global publications: A scientometric study. VSRD International Journal of Library \& Information Science October 2016, 2(5), 67-74.

[27] Liu, Zhongqiu, Yaolin Liu, Yangjie Guo, and Hua Wang. "Progress in Global Parallel Computing Research: a Bibliometric Approach.” Scientometrics 95, no. 3 (December 6, 2012): 967-983. Doi: 10.1007/s11192-012-0927-y.

[28] Dhawan, S.M., Gupta, B.M. and Gupta, R. Global pervasive and ubiquitous computing 2005-14. Annals of Library and Information Studies June 2016, 63(2), 117-25.

[29] Garousi, Vahid. “A Bibliometric Analysis of the Turkish Software Engineering Research Community.” Scientometrics 105, no. 1 (August 12, 2015): 23-49. Doi: 10.1007/s11192-015-1663-x. 